Open Access

\title{
The Anti-Apoptotic Effect of Respiratory Syncytial Virus on Human Peripheral Blood Neutrophils is Mediated by a Monocyte Derived Soluble Factor
}

\author{
Christopher M. Coleman ${ }^{*}$, , Karen Plant ${ }^{1}$, Susan Newton ${ }^{2}$, Lynsey Hobson ${ }^{1}$, Moira K.B. Whyte ${ }^{3}$ and \\ Mark L. Everard ${ }^{4}$ \\ ${ }^{1}$ Academic Unit of Child Health, Stephenson Wing, Sheffield Children's Hospital, Sheffield, S10 2TH, UK \\ ${ }^{2}$ Flow Cytometry Core Facility, School of Medicine and Biomedical Sciences, University of Sheffield, Sheffield. S10 \\ $2 R X, U K$ \\ ${ }^{3}$ Academic Unit of Respiratory Medicine, Department of Infection and Immunity, University of Sheffield Medical School, \\ Beech Hill Road, Sheffield. S10 2RX, UK \\ ${ }^{4}$ Department of Respiratory Medicine, Sheffield Children's Hospital, Western Bank, Sheffield. S10 2TH, UK
}

\begin{abstract}
Respiratory Syncytial Virus (RSV) causes annual epidemics of respiratory disease particularly affecting infants. The associated airway inflammation is characterized by an intense neutrophilia. This neutrophilic inflammation appears to be responsible for much of the pathology and symptoms. Previous work from our group had shown that there are factors within the airways of infants with RSV bronchiolitis that inhibit neutrophil apoptosis. This study was undertaken to determine if RSV can directly affect neutrophil survival.

Neutrophils were isolated from citrated venous blood (collected from healthy adult volunteers) by discontinuous plasma: Percoll gradient centrifugation and, in some experiments, further purified by negative immunomagnetic bead selection. The effect of RSV on neutrophil survival was measured by Annexin V-PE /To-Pro-3 staining and by morphological changes, using Dif-Quick staining of cytospins.

Inhibition of neutrophil apoptosis was observed in neutrophils isolated by standard plasma:Percoll gradient when exposed to RSV but not in ultra pure neutrophil preparations. Adding monocytes back to ultra purified preparations restored the effect. The inhibition of apoptosis was observed with both active and UV inactivated virus. The effect is dependent on a soluble factor and appears to be dependent on CD14 receptors on the monocytes.
\end{abstract}

Keywords: Respiratory syncytial virus, neutrophils, apoptosis, monocytes.

\section{INTRODUCTION}

The human Respiratory Syncytial Virus (hRSV) is responsible for annual epidemics of respiratory disease affecting all age groups throughout the world. It is a particularly important pathogen in infants and young children. In the USA alone it is responsible for over 100,000 infants and young children being admitted to hospital each winter with acute bronchiolitis, pneumonia and exacerbations of asthma [1]. Its importance as a pathogen in the elderly and those with COPD is now well established [2] and it has been suggested that RSV is at least as important a human pathogen as the influenza virus in all but Influenza epidemic years [3].

The inflammation within the airways of infants with RSV infections is dominated by neutrophils with these cells accounting for more than $80 \%$ of cells recovered from both the upper and lower airways [4]. Neutrophils are key cells

*Address correspondence to this author at the Department of Veterinary Medicine, The Ohio State University, Columbus, Ohio, 43201, USA; Tel: 1614-247-8401; Fax: 1-614-292-6473; E-mail: coleman.555@osu.edu within the innate immune response to invading pathogens. Because of the requirement for responding to a range of pathogens, neutrophils carry cytotoxic components which not only kill any invading organism, but can also cause damage to the lungs, either directly or through provocation of an inappropriate inflammatory response [5], hence it is important that they undergo apoptosis (programmed cell death) in a timely manner once activate. Neutrophils in culture undergo spontaneous apoptosis within 24 hours [6], but many immunological and pathogenic factors have been identified which are capable of inhibiting this and prolonging the survival of the cells in vitro [7-11].

Previous work has shown that lavage fluid from the airways of infants with RSV bronchiolitics and, surprisingly, healthy infants contains a soluble factor that is capable of inhibiting the apoptosis of neutrophils [12]. No effect on PMN apoptosis was noted when lavage samples from health adult adults were used. Interestingly a recent study found that there are as yet unidentified factors in induced sputum from adult asthmatics that are able to inhibit PMN apoptosis and that the effect is most marked in the most severely affected individuals [13]. An in vitro study suggested that 
RSV is capable of directly inhibiting the apoptosis of both neutrophils and eosinophils directly in vitro [14]. However this group used conventionally purified granulocytes that frequently contain a small but significant contaminating mononuclear cell population. Recent work has shown that contaminating monocytes contribute to the anti-apoptotic effect of bacterial lipopolysaccharide (LPS) [11, 15], so it is vital that neutrophil populations are ultra-purified prior to analysis of specific effects on apoptosis [15]. Moreover, the purity of the RSV preparation has also been shown to influence the results of in vitro studies in that conventionally purified virus contains contaminating cytokines and other product that can have a significant impact on in vitro experiments [16].

The aims of this study were to determine whether RSV can directly interact with neutrophils to affect their survival and, if not, to explore the potential indirect mechanisms through which RSV might influence neutrophil apoptosis. Since neutrophils are likely to be of major importance during RSV infection, an understanding of the mechanisms driving this process may lead to potential therapeutic interventions designed to limit the inflammatory response.

\section{MATERIALS AND METHODS}

\section{Ethics Statement}

This study involved the use of blood obtained from healthy adult volunteers. The study was considered and approved by The South Sheffield Research Ethics Committee which is associated with the Royal Hallamshire Hospital, Sheffield, UK. Informed written consent was obtained from all volunteers.

\section{Isolation of Polymorphonuclear Leucocytes from Human Blood Using Discontinuous Plasma: Percoll Gradient}

Polymorphonuclear Leucocytes (PMNs; neutrophils, eosinophils and basophils) were isolated from anti-coagulated freshly drawn venous blood from healthy adult volunteers.

PMNs were separated from the peripheral blood mononuclear cells (PBMCs) by discontinuous plasma: Percoll gradient centrifugation [17].

\section{Analysis of Purity of Plasma: Percoll Purified Neutrophils}

Cells were stained with FITC conjugated antibodies to CD3, CD19 and CD56 (all Serotec) or a PE conjugated antiCD66 antibody (BDBiosciences) and analysed by flow cytometry using a FACSCalibur flow cytometer (Beckton Dickinson).

Using flow cytometry analysis of cell surface markers, a typical plasma: Percoll purified neutrophil preparations contained $87 \%( \pm 6.5 \%, \mathrm{n}=6)$ CD66 positive granulocytes, $3 \%( \pm 2 \%, n=4)$ CD3 positive $\mathrm{T}$ cells, $1 \%( \pm 0.5 \%, \mathrm{n}=5)$ CD19 positive B cells, 0.8\% $( \pm 0.6 \%, n=5)$ CD36 positive monocytes and $1 \%(n=2)$ CD56 positive Natural Killer Cells, with any remaining being red blood cells or CD3 negative $\mathrm{T}$ cells.

Assessment of the nature of the granulocytes was done by assessment of cytospin slides. Basophils were never observed in the preparations and eosinophils made up less than $10 \%$ of the cell population.

\section{Ultra Purification of PMNs.}

Ultra-purification of PMNs was performed using negative immunomagnetic selection [11]. Briefly, the resultant PMN layer from the plasma: Percoll gradient was incubated with an antibody cocktail containing antibodies to CD2, CD3, CD19, CD36, CD56 and Glycophorin and magnetic colloid (both StemCell Technologies) and run through a StemSep ${ }^{\mathrm{TM}}$ immunomagnetic column (StemCell Technologies).

All preparations were $99.2( \pm 0.2 \%, n=5)$ CD66 positive granulocytes. The flow cytomery plots of these cells showed very few events outside the granulocyte gates (Fig. 1) and PBMCs were not observed on cytocentrifuge slides. PMNs Basophils were never observed in the preparations and eosinophils made up less than $10 \%$ of the population (by cytocentrifuge slide counts).

\section{Ultra-Purification of Monocytes from the PBMC Layer}

A cocktail containing antibodies to CD2, CD3, CD16, CD19, CD20, CD56, CD66b, CD123 and Glycophorin A along with magnetic colloid (both StemCell Technologies) was added and the cells were purified using the StemSep ${ }^{\mathrm{TM}}$ system (StemCell Technologies).

\section{Add Back Experiments}

Following cell purifications, ultra purified PMNs and monocytes were mixed together in described ratios. The cells were mixed together and aliquoted for infection to ensure even distribution of cells within an experiment.

\section{Depletion of Individual PBMC Populations}

Individual PBMC populations were depleted from the plasma: Percoll purified population by anti-Fluorescein Isothiocyanate (FITC) positive immunomagnetic selection. An anti-CD3 antibody was used to deplete $T$ cell, an antiCD19 antibody to deplete B cells, an anti-CD56 antibody to deplete Natural Killer Cells and both anti-CD14 and antiCD36 antibodies for monocytes.

Cells were depleted using the anti-FITC isolation kit according to the manufacturer's instructions and the EasySepp $^{\mathrm{TM}}$ isolation system (all StemCell technologies). Negative populations were retained and used in experiments.

\section{Maintenance of Cells in Culture}

All cell populations were maintained in RPMI medium (Sigma) supplemented with 10\% Foetal Bovine Serum (FBS; Invitrogen) and 1\% Penicillin and Streptomycin (Sigma).

\section{Identification of Neutrophils on Flow Cytometry Plots}

For analysis, neutrophils are identified on flow cytometry Forward Scatter Side Scatter plots. Representative dot plots of plasma: Percoll purified and ultra-purified populations are shown in Fig. (1). Neutrophils were gated upon, as demonstrated in Fig. (1), for the study of apoptosis in this population exclusively.

\section{Analysis of Apoptosis}

Neutrophil apoptosis was assessed by both Annexin V/ TO-PRO $3^{\mathrm{TM}}$ staining and morphological staining of cytocentrifuge slides. 

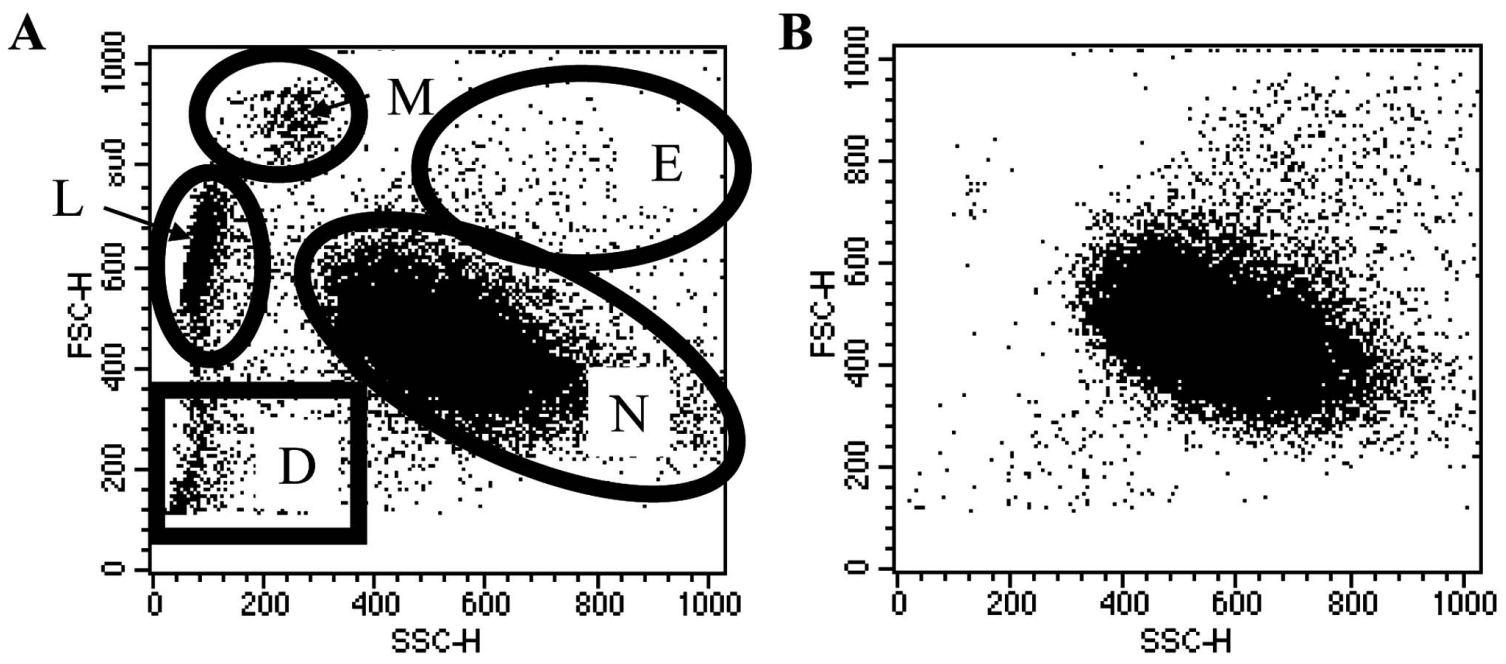

Fig. (1). Representative dot plots of plasma: Percoll (A) and ultra-purified (B) neutrophil populations. Discrete cell populations can be identified, as shown in $\mathbf{A}: \mathbf{M}=$ monocytes; $\mathrm{L}=$ lymphocytes; $\mathrm{N}=$ neutrophils; $\mathrm{E}=$ eosinophils; $\mathrm{D}=$ red blood cells and any cellular debris.

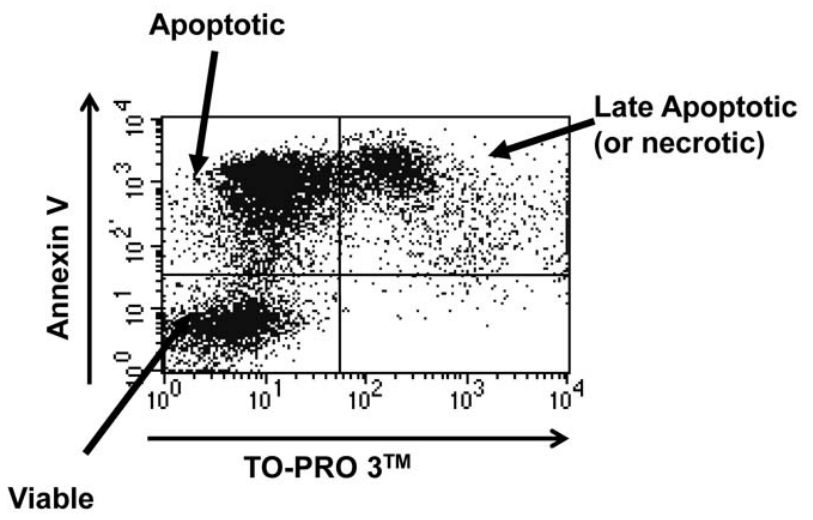

Fig. (2). Representative plot obtained from an AnnexinV/TOPRO $3^{\text {TM }}$ stain on mock infected plasma:Percoll purified neutrophils after 24 hours of culture. Plot shows all 3 possible stains: double negative cells are viable, Annexin V positive only cells are apoptotic and double positive cells are late apoptotic/necrotic.

Following maintenance in culture, cells were harvested and stained with Annexin V conjugated to Phycoerythrin (PE) according to the manufacturer's instructions (BD BioSciences). Just prior to analysis, TO-PRO $3^{\mathrm{TM}}$ (Molecular Probes) was added at a final dilution of 1:10,000 into the sample. Cells were analysed using a FACSCalibur flow cytometer (Beckton Dickinson). Cells were judged to be viable if double negative, early apoptotic if positive for Annexin $\mathrm{V}$ alone and necrotic or late apoptotic if double positive (as shown in Fig. 2). In these experiments significant TO-PRO $3^{\mathrm{TM}}$ positivity was only observed at late time points, so was assumed to be late apoptosis.

Concurrently, cytocentrifuge preparations were made (Cytospin 3; Shandon), fixed in methanol (BDH) and stained with DiffQuick stains (Dade Behring). Slides were examined using oil immersion microscopy and apoptosis was confirmed by characteristic nuclear morphology. The DiffQuick stain stains the nucleus purple. Live neutrophils have a characteristic multi-lobed nucleus with light staining, whilst apoptotic neutrophils have a small, round nucleus with intense purple staining.

\section{RSV Production}

The A2 strain of RSV was propagated in HeLa cells by infection of $90 \%$ confluent cell cultures with A2-RSV and subsequent culture for 2 days. Contaminating cytokines were removed from the A2-RSV containing HeLa cell media using a Vivaspin 20 1,000,000 Dalton pore size (Sartorius, Goettingen, Germany) centrifugation filters as described previously [16]. An immunohistochemical plaque assay for RSV was performed by serial dilution onto HeLa cells and subsequent staining for RSV as described previously [16].

Inactivation of the Virus by Ultra-Violet
Irradiation

(UV)

Virus was made replication deficient by placing it in a Stratalinker. UV Crosslinker (Stratagene, USA) for 10 minutes at full power $\left(\sim 4000 \mu\right.$ watts $\left./ \mathrm{cm}^{2}\right)$.

Non-infectivity of the virus was confirmed by plaque assay.

Testing of Viral Stocks for Lipopolysaccharide Contamination

Virus stocks were tested for LPS contamination using the E-TOXATE $^{\circledR}$ (Sigma) method according to the manufacturer's instructions.

No significant LPS contamination was found in any viral stock used in this study.

\section{Infection of Neutrophil Cultures with RSV}

Freshly isolated cell populations were infected with RSV at a multiplicity of infection (m.o.i.) of $0.5,1,2$ or 10 . The appropriate volume of virus was added immediately after cell isolation to $0.25 \times 10^{6}$ cells in 100 ul total volume and cells were cultured under standard neutrophil culture conditions as described above.

\section{Inhibition of CD14 Signalling}

A known CD14 blocking antibody (clone number MEM18) was used, along with an isotype control (both from Abcam), at a concentration of $10 \mu \mathrm{g} / \mathrm{ml}$. 


\section{Collection and Heat-Inactivation of Monocyte Conditioned Media}

Purified monocytes were challenged with RSV at an m.o.i. of 2 for 12 hours; the media was collected and added to ultra-purified neutrophils at a concentration of 1:1 into the cells. Additionally, the media was heat treated by boiling at $90^{\circ} \mathrm{C}$ for 15 minutes.

\section{Cytokine Bead Array}

Plasma:Percoll pure and ultra-pure neutrophils were incubated with A2-RSV at m.o.i.'s of 2 or 10 for 12 hours. The supernatant was collected, snap-frozen and stored at $80^{\circ} \mathrm{c}$ until 4 sets of samples, from independent donors, were collected. All samples were run at the same time and levels of Interferon- $\gamma$, Tumour Necrosis Factor- $\alpha$, Interleukin (IL)$1 \beta$, IL-6, IL-9, IL-10, IL-12p70, Granulocyte Colony Stimulating Factor, Granulocyte-Macrophage Colony Stimulating Factor, Macrophage Inflammatory Protein (MIP)- $1 \alpha$ and MIP-1 $\beta$ were measured using Cytokine Bead Array kits and a FACSArray Flow Cytometer (Beckton Dickinson). The upper detection limit for each cytokine/chemokine is $5000 \mathrm{pg} / \mathrm{ml}$, the precise lower detection limit varied between cytokines depending on the standard curve, but was always $10-20 \mathrm{pg} / \mathrm{ml}$.

\section{Data Analysis and Statistics}

Data are presented as mean \pm SEM.
Percentage of cells in apoptosis is continuous numerical data that can be considered normally distributed for statistical purposes. To assess the effect of purification on RSV mediated neutrophil apoptosis, where cells are matched according to the donor; data were assessed by two-way ANOVA.

To compare individual treatments or cell populations to each other within the entire data set, the Bonferroni multiple comparison post-test was used.

All statistical analyses were performed using the Minitab statistical analysis software.

Differences were considered to be statistically significant where $\mathrm{p}<0.05$.

\section{RESULTS}

RSV Inhibits the Apoptosis of Peripheral Blood Neutrophils, But Only in the Presence of Contaminating Monocytes

RSV is capable of inhibiting the apoptosis of peripheral blood neutrophils in plasma: Percoll purified cell populations, as shown in Fig. (3). RSV statistically significantly inhibits the apoptosis of plasma: Percoll purified neutrophils at m.o.i.'s of 2 and 10 at 12 and 24 hours post-infection.

When contaminating PBMCs are removed from the cells by ultra-purification, there is a statistically significant attenuation of
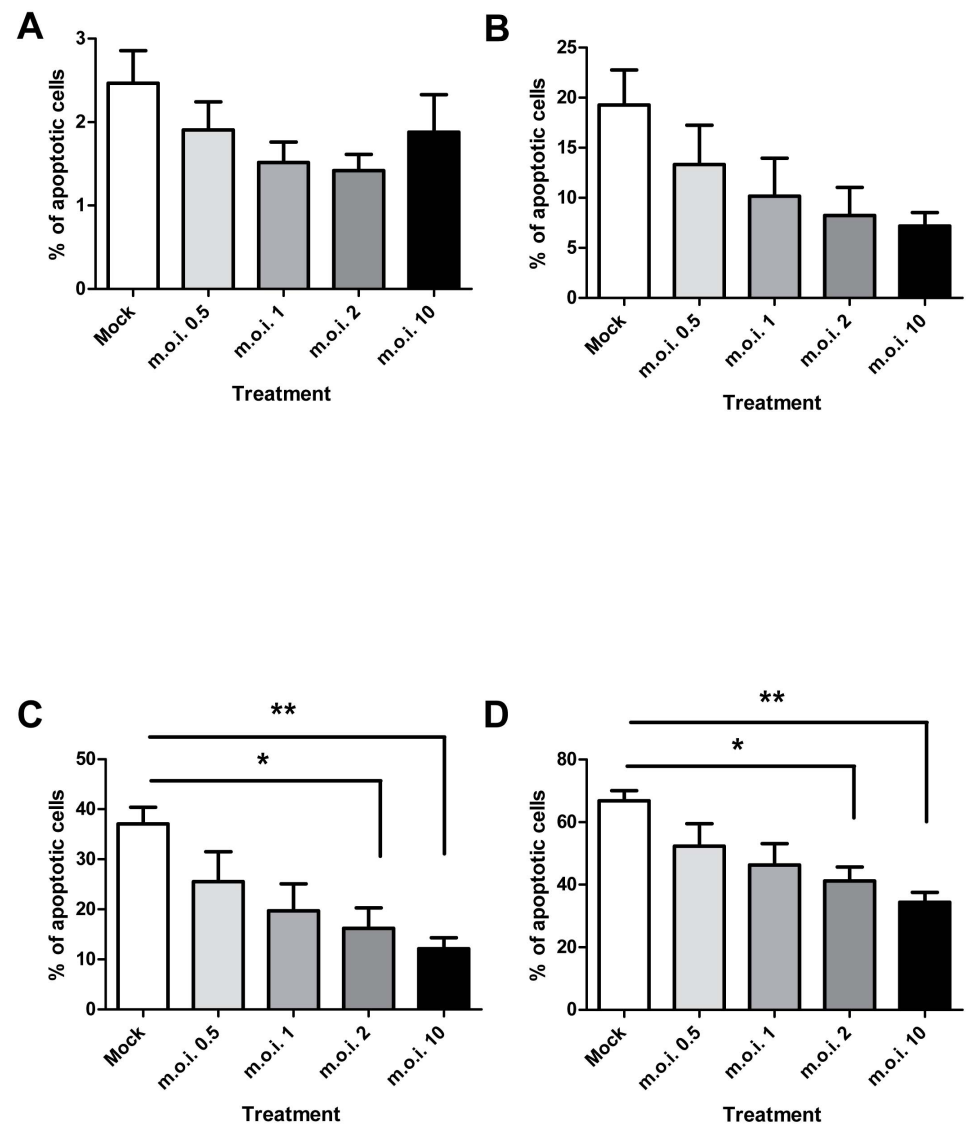

Fig. (3). Effect of RSV on neutrophil apoptosis in plasma: Percoll purified cells at 4 (A), 8 (B), 12 (C) and 24 (D) hours post infection. Data are presented as mean values, with error bars indicating the standard error, of 5 independent experiments on independent donors. Levels of significance are shown as *, $\mathrm{p}<0.05 ; * *, \mathrm{p}<0.01$. 
the anti-apoptotic effect of the virus, as shown in Fig. (4). The 4 hour time point is not shown in Fig. (4), as levels of apoptosis are very low at this time point (Fig. 3A). Cells exposed to virus titres at both 12 and 24 hours post-infection show a statistically significant increase in apoptosis upon removal of the PBMCs (for example, m.o.i. of 2 at 12 hours: plasma: Percoll $v s$ ultra pure $=16.17 \% \pm 4.13 \mathrm{SEM} v s 40.31 \pm 2.89 \mathrm{SEM}$; two-way ANOVA + BONFERRONI: $\mathrm{t}=4.79, \mathrm{p}<0.001)$. Removal of PBMCs had no statistically significant effect on the constitutive apoptosis of mock infected neutrophils.

Typically, ultra-purification of the plasma: Percoll population yielded $50-75 \%$ granulocyte return, which is lower than the CD66 stain implies, thus to confirm that the column purification had no effect on the biology of the neutrophils themselves, or was selectively removing a more RSV responsive neutrophil population, 5\% donor matched mixed PBMCs were added back to ultra-purified populations and then infected with RSV. The RSV mediated inhibition of neutrophil apoptosis was restored in these populations (data not shown).

When CD14 (a monocye marker) depletion experiments were performed, the anti-apoptotic effect of the RSV was statistically significantly attenuated, as shown in Fig. (5). For all m.o.i.'s of the virus, there is a statistically significant effect of removing the CD14 positive population on apoptosis of neutrophils (for example, at m.o.i. of 2: plasma: Percoll pure $v s$ CD14 depleted $=9.15 \% \pm 3.08$ SEM vs 21.37 \pm 0.98 SEM; $p<0.05)$. Removal of the CD14 positive population has no statistically significant effect on constitutive apoptosis (mock infected controls). Furthermore, depletion of CD3 (T Cells), CD19 (B Cells) and CD56 (Natural Killer Cells) showed no effect on the RSV mediated inhibition of neutrophil apoptosis (Table 1).

When ultra-purified monocytes are added back to ultrapurified neutrophils there is a restoration of the RSV mediated anti-apoptotic effect, as shown in Fig. (6). Though addition of $5 \%$ monocytes statistically significantly inhibits constitutive neutrophil apoptosis, there is a much larger and more statistically significant difference between the populations in the presence of all RSV doses (for example, at the m.o.i. of 2: ultra pure vs $5 \%$ add-back $=51.53 \% \pm 7.37$ SEM vs $33.58 \pm 7.59$ SEM; two-way ANOVA + BONFERRONI: $\mathrm{t}=5.08, \mathrm{p}<0.001) .1 \%$ monocyte add-back is not sufficient to restore the anti-apoptotic effect, though, at the m.o.i. of 2 , in particular, there is evidence of a dose dependent effect of the monocytes $(51.53 \%$ vs $44.04 \%$ vs $33.58 \%$ for mock, $1 \%$ and $5 \%$ respectively).

\section{CD14 is Required for the Anti-Apoptotic Effect}

When a CD14 blocking antibody was added to plasma: Percoll purified cell cultures, the anti-apoptotic effect of RSV was statistically significantly attenuated as shown in Fig. (7).

The Virus Does Not have to be Fully Infectious to have its Anti-Apoptotic Effect

When UV-inactivated A2-RSV is added to plasma: Percoll purified neutrophils with concurrent untreated controls (both at an m.o.i. of 2), there is no statistically significant effect on the RSV mediated inhibition of neutrophil apoptosis, as shown in Fig. (8).
A

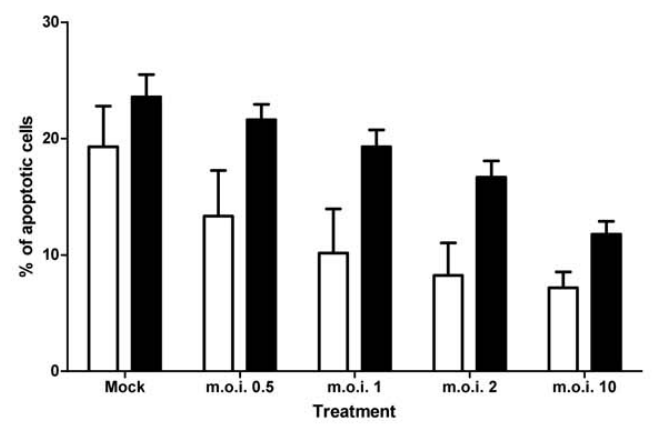

B
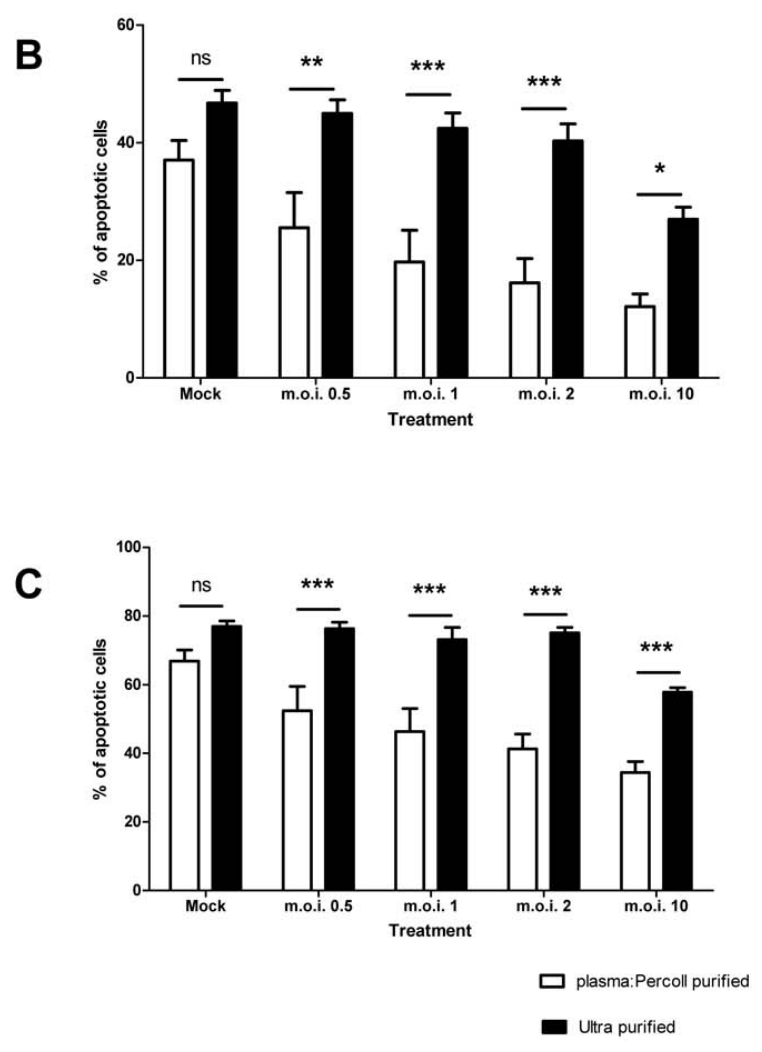

Fig. (4). Effect of RSV on neutrophil apoptosis in plasma: Percoll purified and ultra purified cells at 8 (A), 12 (B) and 24 (C) hours post infection. Data are presented as mean values, with error bars indicating the standard error, of 5 independent experiments on independent donors. Levels of significance are shown as $*, \mathrm{p}<0.05 ; * *, \mathrm{p}<0.01 ; * * *, \mathrm{p}<0.001 ; \mathrm{ns}=$ not statistically significant.

Monocytes Challenged with RSV Produce a Heat Labile, Soluble Factor which Blocks Neutrophil Apoptosis

The media from RSV treated monocytes statistically significantly inhibited neutrophil apoptosis as compared to untreated monocyte media $(\mathrm{p}<0.01)$ and this effect can be removed by heat treatment $(\mathrm{p}<0.01)$, as shown in Fig. (9).

\section{Cytokine and Chemokine Analysis}

In an effort to determine if there was a particular cytokine or chemokine responsible for the anti-apoptotic effect, a cytokine bead array was performed on samples from 
Table 1. Representative Data of the Effect of Depletion of CD3 Positive, CD19 Positive and CD56 Positive Cells from Plasma: Percoll Purified Neutrophil Populations. Cells were Infected with RSV at an m.o.i. of 2 and Apoptosis was Assessed at 12 Hours Post-Infection. Experiments were Performed at Least Twice on Cells from Different Donors

\begin{tabular}{|c|c|c|c|}
\hline $\begin{array}{c}\text { Depletion } \\
\text { Performed. }\end{array}$ & $\begin{array}{c}\text { Donor Matched Plasma: Percoll Purified Neutrophils } \\
\text { (\% of Apoptotic Cells) }\end{array}$ & $\begin{array}{c}\text { Donor Matched Ultra Purified Neutrophils } \\
\text { (\% of Apoptotic Cells) }\end{array}$ & $\begin{array}{c}\text { Depleted Cell Population } \\
\text { (\%of Apoptotic Cells) }\end{array}$ \\
\hline \hline CD3 & $6.34 \%$ & $30.90 \%$ & $9.2 \%$ \\
\hline CD19 & $16.37 \%$ & $31.58 \%$ & $18.06 \%$ \\
\hline CD56 & $15.33 \%$ & $26.67 \%$ & $6.00 \%$ \\
\hline
\end{tabular}

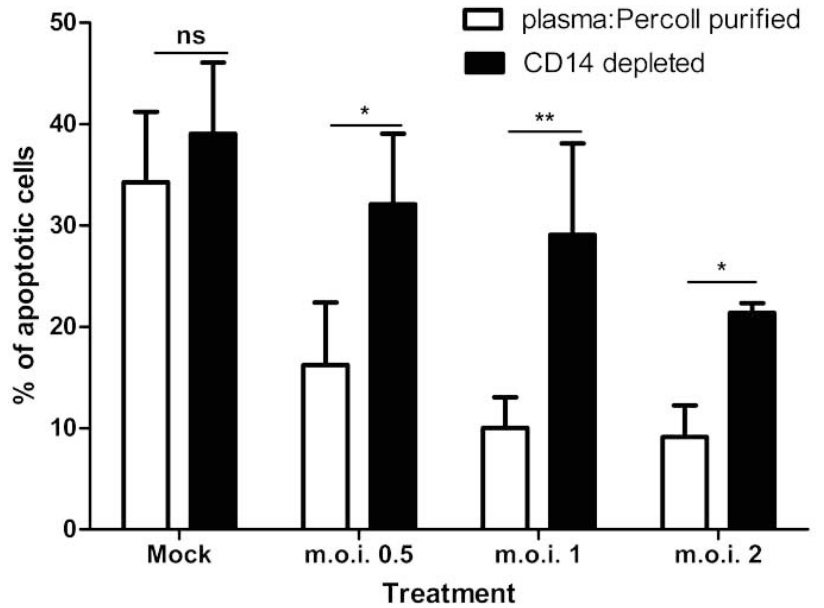

Fig. (5). Effect of RSV on neutrophil apoptosis in a CD14 depleted cell population at 12 hours post-infection. Data are presented as mean values, with error bars indicating the standard error, of 3 independent experiments on independent donors. Data was analysed by two way ANOVA and the Bonferroni post-test. Levels of significance are shown as ${ }^{* *}, \mathrm{p}<0.01 ;{ }^{*}, \mathrm{p}<0.05$. ns $=$ not statistically significant.

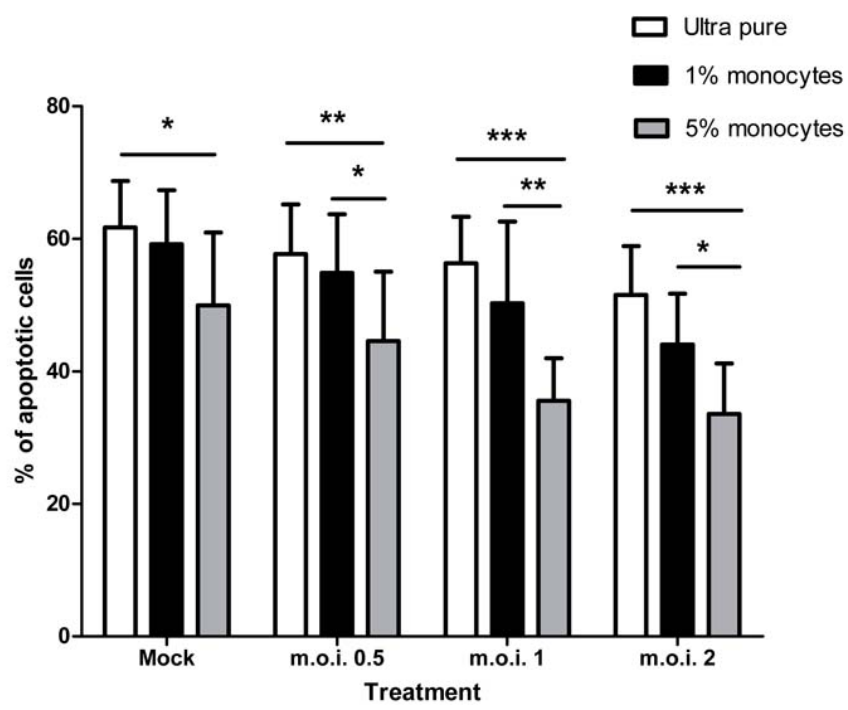

Fig. (6). Effect of RSV on neutrophil apoptosis in ultra purified neutrophils with $1 \%$ and $5 \%$ monocyte add-backs at 12 hour time point. Data are presented as mean values, with error bars indicating the standard error, of 3 independent experiments on independent donors. Data was analysed by two way ANOVA and the Bonferroni post-test. Levels of significance are shown as *, $\mathrm{p}<0.05 ; * *, \mathrm{p}<0.01 ; * * *, \mathrm{p}<0.001$.

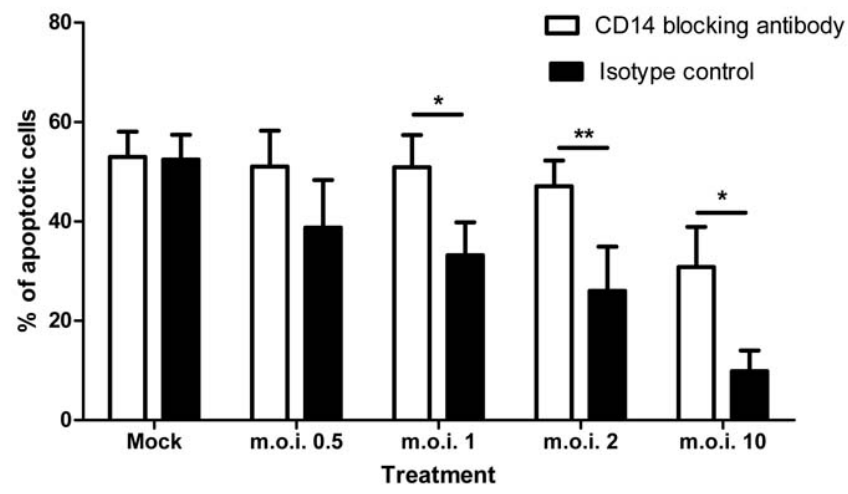

Fig. (7). Effect of a CD14 blocking antibody, or the relevant isotype control on RSV inhibition of neutrophil apoptosis at the stated m.o.i.'s at 12 hours post-infection. Data are presented as mean values, with error bars indicating the standard error, of 3 independent experiments on independent donors. Data was analysed by two way ANOVA and the Bonferroni post-test. Levels of significance are shown as ${ }^{* *}, \mathrm{p}<0.01 ;{ }^{*}, \mathrm{p}<0.05$.

both plasma: Percoll and ultra purified cells challenged with RSV at an m.o.i. of 2 or 10 , the results of which are shown in Tables 2 and 3 respectively. The cells were treated the same as in all other experiments, therefore the cytokine/chemokine values shown were produced from $0.25 \times 10^{6}$ cells treated with RSV. A 12 hour time point was chosen as a time point, as this point shows significant inhibition of neutrophil apoptosis (Fig. 3) and a significant difference between plasma:Percoll purified and ultra purified cell populations (Fig. 4).

These data show that a range of pro-inflammatory cytokines and chemokines are upregulated in plasma:Percoll purified cells in response to RSV in a dose dependent manner. However, in ultra-purified cell populations, only MIP- $1 \alpha$ and MIP-1 $\beta$ are significantly upregulated in a dose dependent manner.

Following on from these results, the effects of recombinant MIP-1 $\alpha$ and MIP-1 $\beta$ (R\&D Technologies) were used to assess if either of these chemokines are capable if inhibiting neutrophil apoptosis. Neither had any effect at any concentration tested (data not shown).

\section{DISCUSSION}

The results from this study would indicate that the Respiratory Syncytial Virus does not directly influence the survival of neutrophils. However, one or more soluble mediators released by monocytes exposed to RSV do have a statistically significant impact on neutrophil survival. These 
Table 2. Cytokine and Chemokine Levels, Measured in pg/ml, in Plasma: Percoll Purified Cell Population in Presence or Absence of A2-RSV

\begin{tabular}{|c|c|c|c|}
\hline & Mock & A2-RSV (m.o.i. 2) & A2-RSV (m.o.i. 10) \\
\hline \hline IFN- $\gamma$ & 14.16 & BDL & BDL \\
\hline TNF- $\alpha$ & 2.83 & $66.55 \pm 19.86$ & $113.57 \pm 23.56$ \\
\hline IL-1 $\beta$ & 5.04 & $60.85 \pm 19.62$ & $1456.74 \pm 714.64$ \\
\hline IL-6 & BDL & $370.78 \pm 211.30$ & $17.18 \pm 10.55$ \\
\hline IL-9 & 27.09 & $13.66 \pm 13.66$ & $15.02 \pm 4.84$ \\
\hline IL-10 & 2.71 & $6.76 \pm 2.96$ & BDL \\
\hline IL-12p70 & BDL & $5.01 \pm 5.01$ & $18.76 \pm 6.39$ \\
\hline G-CSF & BDL & $3.44 \pm 3.44$ & $53.38 \pm 22.71$ \\
\hline GM-CSF & 4.03 & $765.37 \pm 199.18$ & $2.61 \pm 2.61$ \\
\hline MIP-1 $\alpha$ & 2.69 & ADL & $3823.72 \pm 855.41$ \\
\hline MIP-1 $\beta$ & 70.00 & ADL \\
\hline
\end{tabular}

Data is mean values $+/$ - standard error of 4 independent experiments. Mock infected controls are mean values of 2 independent experiments. BDL $=$ below detection limit; ADL $=$ above detection limit.

Table 3. Cytokine and Chemokine Levels, Measured in pg/ml, in the Ultra Purified Cell Population in Presence or Absence of A2RSV

\begin{tabular}{|c|c|c|c|}
\hline & Mock & A2-RSV (m.o.i. 2) & A2-RSV (m.o.i. 10) \\
\hline \hline IFN- $\gamma$ & BDL & BDL & $1.53 \pm 1.53$ \\
\hline TNF- $\alpha$ & BDL & $2.46 \pm 1.50$ & $2.33 \pm 2.33$ \\
\hline IL-1 $\beta$ & BDL & $2.73 \pm 1.63$ & $16.40 \pm 5.05$ \\
\hline IL-6 & BDL & $7.91 \pm 2.29$ & $3.99 \pm 3.99$ \\
\hline IL-9 & 24.43 & $7.15 \pm 7.15$ & BDL \\
\hline IL-10 & BDL & $2.65 \pm 1.53$ & $2.78 \pm 2.78$ \\
\hline IL-12p70 & BDL & BDL & $4.71 \pm 4.71$ \\
\hline G-CSF & BDL & BDL & $2.67 \pm 2.67$ \\
\hline GM-CSF & BDL & BDL & $114.69 \pm 46.45$ \\
\hline MIP-1 $\alpha$ & BDL & $25.21 \pm 4.47$ & $3307.50 \pm 865.19$ \\
\hline MIP-1 $\beta$ & BDL & $816.00 \pm 147.60$ & \\
\hline
\end{tabular}

Data is shown as mean values $+/$ - standard error of 4 independent experiments. Mock infected controls are mean values of 2 independent experiments. BDL $=$ below detection limit.

results are consistent with our previous results which indicated that RSV does not directly activate neutrophils in vitro [16]. The results contrast with a number of other published studies which have suggested a number of interactions between virus and neutrophils $[12,14,16,18$ $21]$, but is of note that all of these studies have potential problems regarding the purity of virus [16] and/or neutrophils [15].

The importance of ultra-purifying neutrophil populations in order to assess the direct effects of factors upon neutrophil biology has been demonstrated in the context of the effects of LPS on neutrophil survival [11]. Ultra-purification of neutrophils has no significant effect on the fundamental biology of the neutrophils, with cells still able to respond to LPS and no difference in TLR expression patterns [11], though PBMCs do inhibit neutrophil apoptosis by their presence in the absence of other stimuli [15]. It has also been shown that LPS has a significantly greater effect on inhibiting neutrophil apoptosis in the presence of the small numbers of contaminating monocytes present in standard purified neutrophil populations [11], indicating the importance of using highly purified neutrophil populations to study specific interactions with pathogenic stimuli [15].

In the specific case of the effect of RSV on neutrophil apoptosis, one study appeared to indicate that RSV directly inhibits the apoptosis of neutrophils [11] but they use a PMN population similar to that purified by plasma: Percoll gradient in this study and it is now clear from the results from the current study that the purity of the PMN population is of vital importance to the study of RSV interactions with neutrophils when undertaking in vitro studies. 

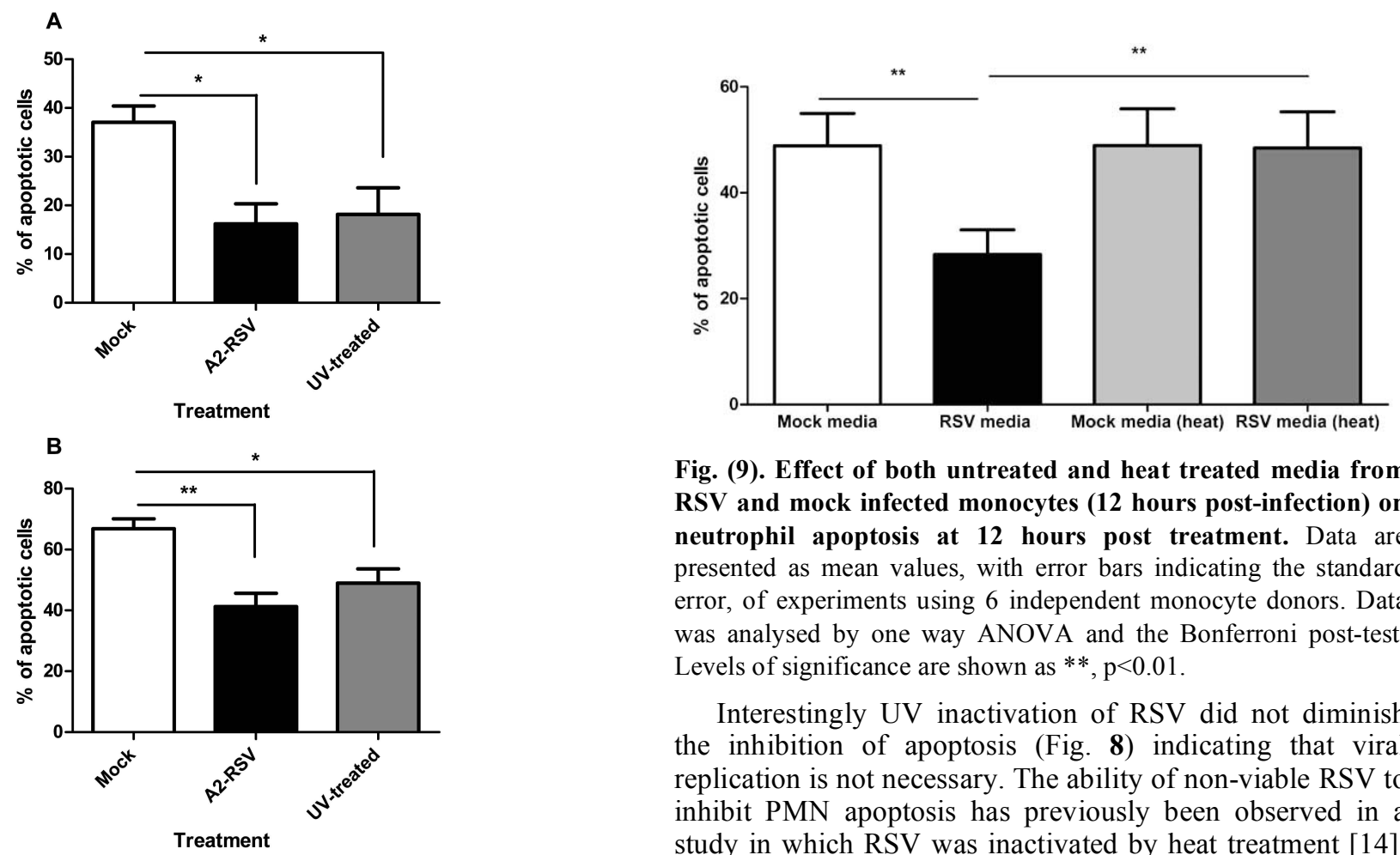

Fig. (9). Effect of both untreated and heat treated media from RSV and mock infected monocytes (12 hours post-infection) on neutrophil apoptosis at 12 hours post treatment. Data are presented as mean values, with error bars indicating the standard error, of experiments using 6 independent monocyte donors. Data was analysed by one way ANOVA and the Bonferroni post-test. Levels of significance are shown as **, $\mathrm{p}<0.01$.

Interestingly UV inactivation of RSV did not diminish the inhibition of apoptosis (Fig. 8) indicating that viral replication is not necessary. The ability of non-viable RSV to inhibit PMN apoptosis has previously been observed in a study in which RSV was inactivated by heat treatment [14]. These data suggest that a viral structural protein (or the viral RNA) is responsible for the effect. A knock out mutant of the membrane Glycoprotein was utilised but showed no attenuation in this model (data not shown).

Blocking CD14 mediated signalling in these cultures statistically significantly attenuated the anti-apoptotic effect of RSV (Fig. 7), implying a role for Toll Like Receptors (TLRs). Both TLR4 [22], TLR3 [23] and TLR2 complexes [24-27] are known to complex with CD14. A number of studies have attempted to determine whether there is a direct interaction between RSV and TLR4. The evidence is conflicting with some papers suggesting a role for this molecule in RSV pathogenesis [28-30] and others suggesting that there is no direct interaction [14, 31]. Kurt-Jones et al. showed that TLR4/CD14 complexes and interactions with the RSV F protein are vitally important for RSV clearance [30], though it has since been suggested that this observation is a result of the mouse strain utilised in these experiments, which also has a knock-out in the Interleukin 12 receptor [31]. In the context of RNA virus infection, the interaction between TLR3 and CD14 may be important [23]. RSV has been shown to cause upregulation of TLR3 in infected airways [32] and interactions between RSV and TLR3 cause pro-inflammatory cytokine release from epithelial cells [33], though no studies have investigated this effect in monocytes or other immune cells. A recent study has suggested that the TLR2/TLR6 heterodimeric complex may be involved in RSV pathogenesis. RSV infection of TL2/TLR6 knockout mice was associated with decreased cytokine production, neutrophil recruitment and dendritic cell activation [34]. The exact role of CD14 signalling in monocytes in the pathogenesis of the disease and indeed in neutrophil survival remains to be determined.

Neutrophils themselves express a low level of CD14 on the cell surface [35], so it is possible that the CD14 blocking 
antibody is also blocking CD14 on the neutrophils and inhibiting the RSV mediated inhibition of apoptosis. The antibody cocktail used to ultra-purify the neutrophils does not contain a CD14 antibody and it has been shown that even when such an antibody is used to purify the cells, there is no significant effect on neutrophils [36]. Thus, the CD14 mediated signalling pathway will not be blocked in the ultrapurified cultures and RSV still does not inhibit apoptosis in these cultures; however, we cannot exclude that possibility that the high dose RSV inhibits neutrophil apoptosis via CD14 on the neutrophils.

We were able to demonstrate that monocytes produce a heat labile factor in response to RSV challenge, which is capable of inhibiting neutrophil apoptosis (Fig. 9). In order to assess whether this factor was a cytokine/chemokine, we assessed production of a number of these by Cytokine Bead Array in the plasma: Percoll and ultra-purified cell population. A number of cytokines and chemokines are upregulated in plasma: Percoll purified samples (Table 2). However in the ultra purified samples (Table 3) most of these cytokines were not present in statistically significant quantities. Though levels of MIP- $1 \alpha$ and MIP- $1 \beta$ were reduced 10 fold in the ultra-purified preparations they were still present in statistically significant quantities supporting the suggestion that an extremely small number of residual monocytes are probably still present.

We were unable to positively identify the factor responsible for the anti-apoptotic effect of RSV and finding such a factor will be a significant undertaking, as many host factors are known to inhibit neutrophil apoptosis [7-9, 12]. It remains possible that no single factor is responsible and there is a synergistic relationship between a number of factors, indeed Tables $\mathbf{2}$ and $\mathbf{3}$ show up-regulation of a number of known anti-apoptotic cytokines (IL-1 $\beta$, IL-6 and TNF- $\alpha$ ). Previous attempts to identify the soluble heat labile factor(s) in airways lining fluid from infants with RSV infection that in airways lining fluid using inhibitors against the most likely candidates also failed to identify a single mediator [12] supporting the suggestion that a number of factors are involved. A recent publication using induced sputum samples from asthmatic subjects observed a similar effect on PMN survival but they too were unable to identify the factor(s) responsible [13]. The similarity in results reported when using samples from asthmatics and from infants with an acute viral respiratory tract infection would suggest that the inhibition of PMN apoptosis is a not specific to neither respiratory viral infections nor asthma.

The importance of monocytes in mediating the inhibition of neutrophil apoptosis is perhaps not surprising given their importance in generating cytokines and other factors in response to RSV [37-44]. The significance of the inhibition of neutrophil apoptosis in the pathogenesis of the disease is unclear. There does seem to be little doubt that the intense neutrophilia observed in the airways of infants with acute bronchiolitis contribute significantly to symptoms and disease severity through the release of products such as human neutrophil elastase and myeloperoxidase which induce mucus secretion, airways oedema and cough. Indeed this inflammatory response with considerable amounts of airways secretions in both the upper and lower airways together with coughing and sneezing would appear to be ideally suited to transmission of the virus. To date there is no evidence that the intense neutrophil response observed in primary infection in infants contributes to eliminating or indeed limiting RSV infection. However, it remains possible that neutrophils do have a role in vivo in eliminating virus either in the presence of antibody attached to the virus or following neutrophil activation

These experiments indicate that the inhibition of neutrophil apoptosis by RSV observed in vitro and in vivo is secondary to activation of monocytes, which produce a heat labile soluble factor(s) and that this process is initiated in response to one or more structural proteins or viral RNA. This study, and our previous study indicating that RSV does not directly activate neutrophils [16], would suggest that the Respiratory Syncytial Virus does not interact directly with neutrophils.

\section{ACKNOWLEDGEMENTS}

The authors would like to acknowledge all members of the Department of Respiratory Medicine at the University of Sheffield, UK for their helpful advice and support throughout this study. Specifically, we would like to thank Professor Ian Sabroe and his laboratory for help and advice regarding neutrophil preparations. $\mathrm{CC}$ and the study were supported by the Sheffield Children's Hospital Research Charity

\section{REFERENCES}

[1] Everard ML. Respiratory Syncytial Virus Bronchiolitis and Pneumonia. In: Taussig LM, Landau L, Eds. Textbook of paediatric medicine. $2^{\text {nd }}$ ed. St Louis: Mosb 2009; pp. 491-500.

[2] Falsey AR, Hennessey PA, Formica MA, Cox C, Walsh EE. Respiratory syncytial virus infection in elderly and high-risk adults. N Engl J Med 2005; 352(17): 1749-59.

[3] Fleming DM, Cross KW. Respiratory syncytial virus or influenza? Lancet 1993; 342(8886-8887): 1507-10.

[4] Everard ML, Swarbrick A, Wrightham M, et al. Analysis of cells obtained by bronchial lavage of infants with respiratory syncytial virus infection. Arch Dis Child 1994; 71(5): 428-32.

[5] Vermes I, Haanen C. Apoptosis and programmed cell death in health and disease. Adv Clin Chem 1994; 31: 177-246.

[6] Haslett C, Savill JS, Whyte MK, Stern M, Dransfield I, Meagher LC. Granulocyte apoptosis and the control of inflammation. Philos Trans R Soc Lond B Biol Sci 1994; 345(1313): 327-33.

[7] Mecklenburgh KI, Walmsley SR, Cowburn AS, et al. Involvement of a ferroprotein sensor in hypoxia-mediated inhibition of neutrophil apoptosis. Blood 2002; 100(8): 3008-16.

[8] Vaughan KR, Stokes L, Prince LR, et al. Inhibition of neutrophil apoptosis by ATP is mediated by the P2Y11 receptor. J Immunol 2007; 179(12): 8544-53.

[9] Lee A, Whyte MK, Haslett C. Inhibition of apoptosis and prolongation of neutrophil functional longevity by inflammatory mediators. J Leukoc Biol 1993; 54(4): 283-8.

[10] Skarman PJ, Rahbar A, Xie X, Soderberg-Naucler C. Induction of polymorphonuclear leukocyte response by human cytomegalovirus. Microbes Infect 2006; 8(6): 1592-601.

[11] Sabroe I, Jones EC, Usher LR, Whyte MK, Dower SK. Toll-like receptor (TLR)2 and TLR4 in human peripheral blood granulocytes: a critical role for monocytes in leukocyte lipopolysaccharide responses. J Immunol 2002; 168(9): 4701-10.

[12] Jones A, Qui JM, Bataki E, et al. Neutrophil survival is prolonged in the airways of healthy infants and infants with RSV bronchiolitis. Eur Respir J 2002; 20(3): 651-7.

[13] Uddin M, Nong G, Ward J, et al. Prosurvival activity for airway neutrophils in severe asthma. Thorax 2010; 65(8): 684-9.

[14] Lindemans CA, Coffer PJ, Schellens IM, de Graaff PM, Kimpen JL, Koenderman L. Respiratory syncytial virus inhibits granulocyte apoptosis through a phosphatidylinositol 3-kinase and NF-kappaBdependent mechanism. J Immunol 2006; 176(9): 5529-37. 
[15] Sabroe I, Prince LR, Dower SK, Walmsley SR, Chilvers ER, Whyte MK. What can we learn from highly purified neutrophils? Biochem Soc Trans 2004; 32(Pt3): 468-9.

[16] Bataki EL, Evans GS, Everard ML. Respiratory syncytial virus and neutrophil activation. Clin Exp Immunol 2005; 140(3): 470-7.

[17] Haslett C, Guthrie LA, Kopaniak MM, Johnston RB Jr, Henson PM. Modulation of multiple neutrophil functions by preparative methods or trace concentrations of bacterial lipopolysaccharide. Am J Pathol 1985; 119(1): 101-10.

[18] Arnold R, Werner F, Humbert B, Werchau H, Konig W. Effect of respiratory syncytial virus-antibody complexes on cytokine (IL-8, IL-6, TNF-alpha) release and respiratory burst in human granulocytes. Immunology 1994; 82(2): 184-91.

[19] Jaovisidha P, Peeples ME, Brees AA, Carpenter LR, Moy JN. Respiratory syncytial virus stimulates neutrophil degranulation and chemokine release. J Immunol 1999; 163(5): 2816-20.

[20] Konig B, Krusat T, Streckert HJ, Konig W. IL-8 release from human neutrophils by the respiratory syncytial virus is independent of viral replication. J Leukoc Biol 1996; 60(2): 253-60.

[21] Stark JM, Godding V, Sedgwick JB, Busse WW. Respiratory syncytial virus infection enhances neutrophil and eosinophil adhesion to cultured respiratory epithelial cells. Roles of CD18 and intercellular adhesion molecule-1. J Immunol 1996; 156(12): 477482.

[22] Chow JC, Young DW, Golenbock DT, Christ WJ, Gusovsky F. Toll-like receptor-4 mediates lipopolysaccharide-induced signal transduction. J Biol Chem 1999; 274(16): 10689-92.

[23] Lee HK, Dunzendorfer S, Soldau K, Tobias PS. Double-stranded RNA-mediated TLR3 activation is enhanced by CD14. Immunity 2006; 24(2): 153-63.

[24] Bas S, Neff L, Vuillet M, et al. The proinflammatory cytokine response to Chlamydia trachomatis elementary bodies in human macrophages is partly mediated by a lipoprotein, the macrophage infectivity potentiator, through TLR2/TLR1/TLR6 and CD14. J Immunol 2008; 180(2): 1158-68.

[25] Bsibsi M, Bajramovic JJ, Van Duijvenvoorden E, et al. Identification of soluble CD14 as an endogenous agonist for Tolllike receptor 2 on human astrocytes by genome-scale functional screening of glial cell derived proteins. Glia 2007; 55(5): 473-82.

[26] Janot L, Secher T, Torres D, et al. CD14 works with toll-like receptor 2 to contribute to recognition and control of Listeria monocytogenes infection. J Infect Dis 2008; 198(1): 115-24.

[27] Nakata T, Yasuda M, Fujita M, et al. CD14 directly binds to triacylated lipopeptides and facilitates recognition of the lipopeptides by the receptor complex of Toll-like receptors 2 and 1 without binding to the complex. Cell Microbiol 2006; 8(12): 1899909.

[28] Tal G, Mandelberg A, Dalal I, et al. Association between common Toll-like receptor 4 mutations and severe respiratory syncytial virus disease. J Infect Dis 2004; 189(11): 2057-63.

[29] Haynes LM, Moore DD, Kurt-Jones EA, Finberg RW, Anderson LJ, Tripp RA. Involvement of toll-like receptor 4 in innate immunity to respiratory syncytial virus. J Virol 2001; 75(22): 10730-7.
[30] Kurt-Jones EA, Popova L, Kwinn L, et al. Pattern recognition receptors TLR4 and CD14 mediate response to respiratory syncytial virus. Nat Immunol 2000; 1(5): 398-401.

[31] Ehl S, Bischoff R, Ostler T, et al. The role of Toll-like receptor 4 versus interleukin-12 in immunity to respiratory syncytial virus. Eur J Immunol 2004; 34(4): 1146-53.

[32] Groskreutz DJ, Monick MM, Powers LS, Yarovinsky TO, Look DC, Hunninghake GW. Respiratory syncytial virus induces TLR3 protein and protein kinase R, leading to increased double-stranded RNA responsiveness in airway epithelial cells. J Immunol 2006; 176(3): 1733-40.

[33] Rudd BD, Burstein E, Duckett CS, Li X, Lukacs NW. Differential role for TLR3 in respiratory syncytial virus-induced chemokine expression. J Virol 2005; 79(6): 3350-7.

[34] Murawski MR, Bowen GN, Cerny AM, et al. RSV Activates Innate Immunity through Toll-like Receptor 2. J Virol 2009; 83(3): 1492500.

[35] Antal-Szalmas P, Strijp JA, Weersink AJ, Verhoef J, Van Kessel KP. Quantitation of surface CD14 on human monocytes and neutrophils. J Leukoc Biol 1997; 61(6): 721-8.

[36] Sabroe I, Prince LR, Jones EC, et al. Selective roles for Toll-like receptor (TLR)2 and TLR4 in the regulation of neutrophil activation and life span. J Immunol 2003; 170(10): 5268-75.

[37] Takeuchi R, Tsutsumi H, Osaki M, Sone S, Imai S, Chiba S. Respiratory syncytial virus infection of neonatal monocytes stimulates synthesis of interferon regulatory factor 1 and interleukin-1beta (IL-1beta)-converting enzyme and secretion of IL-1beta. J Virol 1998; 72(1): 837-40.

[38] Tripp RA, Moore D, Barskey At, et al. Peripheral blood mononuclear cells from infants hospitalized because of respiratory syncytial virus infection express $\mathrm{T}$ helper-1 and $\mathrm{T}$ helper-2 cytokines and CC chemokine messenger RNA. J Infect Dis 2002; 185(10): 1388-94.

[39] Becker S, Quay J, Soukup J. Cytokine (tumor necrosis factor, IL-6, and IL-8) production by respiratory syncytial virus-infected human alveolar macrophages. J Immunol 1991; 147(12): 4307-12.

[40] Kauth M, Grage-Griebenow E, Rohde G, et al. Synergistically upregulated interleukin-10 production in cocultures of monocytes and $\mathrm{T}$ cells after stimulation with respiratory syncytial virus. Int Arch Allergy Immunol 2007; 142(2): 116-26.

[41] Konig B, Streckert HJ, Krusat T, Konig W. Respiratory syncytial virus G-protein modulates cytokine release from human peripheral blood mononuclear cells. J Leukoc Biol 1996; 59(3): 403-6.

[42] Krilov LR, McCloskey TW, Harkness SH, Pontrelli L, Pahwa S. Alterations in apoptosis of cord and adult peripheral blood mononuclear cells induced by in vitro infection with respiratory syncytial virus. J Infect Dis 2000; 181(1): 349-53.

[43] Morrison PT, Thomas LH, Sharland M, Friedland JS. RSV-infected airway epithelial cells cause biphasic up-regulation of CCR1 expression on human monocytes. J Leukoc Biol 2007; 81(6): 148795.

[44] Panuska JR, Merolla R, Rebert NA, et al. Respiratory syncytial virus induces interleukin-10 by human alveolar macrophages. Suppression of early cytokine production and implications for incomplete immunity. J Clin Invest 1995; 96(5): 2445-53. 